\title{
reciturands
}

Revista Científica Mundo de la Investigación y el Conocimiento

Lorena Valverde Palma ${ }^{\text {a }}$; Erick Reyes Ramos ${ }^{\text {b; }}$ Carlota Palma Estrada ${ }^{\text {c }}$ J Janet Paola Emén Sánchez ${ }^{\text {d; }}$ Manuel Bernardo Balladares Mazzini ${ }^{\text {e }}$

Influencia del estado nutricional en el rendimiento escolar de estudiantes de Educación General Básica Media

Influence of nutritional status on the school performance of students of General Basic Basic Education

Revista Científica Mundo de la Investigación y el Conocimiento. Vol. 3 núm. 4., diciembre, ISSN: 2588-073X, 2019, pp. 528-548

DOI: $10.26820 /$ recimundo/3.(4).diciembre.2019.528-548

URL: http://recimundo.com/index.php/es/article/view/673

Código UNESCO: 3205 Medicina Interna

Tipo de Investigación: Artículo de Revisión

(c) RECIMUNDO; Editorial Saberes del Conocimiento, 2019

Recibido: 15/09/2019 Aceptado: 23/11/2019Ｐublicado: 30/12/2019

Correspondencia: karinaborjaa@gmail.com

a. Médico General; Investigadora de Proyecto Semillero; FCM; Universidad de Guayaquil; Guayaquil, Ecuador; lore_13_1_92@hotmail.com

b. Médico General; Investigador de Proyecto Semillero; FCM; Universidad de Guayaquil; Guayaquil, Ecuador; e_reyes_r@hotmail.com

c. Especialista en Pediatría; Magíster en Nutrición; Profesora Principal de la Carrera de Medicina; FCM; Universidad de Guayaquil; Guayaquil, Ecuador; carlota.palmae@ug.edu.ec

d. Especialista en Medicina del Deporte; Master Universitario en Nutrición Humana y Dietética Aplicada; Profesora Titular de la Carrera de Medicina; FCM; Universidad de Guayaquil; Guayaquil, Ecuador; janet.emens@ug.edu.ec

e. Especialista en Medicina del Deporte; Magister en Nutrición; Magister en Salud Pública; Diploma Superior en Diseño Curricular por Competencias; Profesor Titular de la Carrera de Medicina; FCM; Universidad de Guayaquil; Guayaquil, Ecuador; manuel.balladaresm@ug.edu.ec 


\section{Influencia del estado nutricional en el rendimiento escolar de estudiantes de Educación General Básica Media}

Vol. 3, núm. 4., (2019) Lorena Valverde Palma; Erick Reyes Ramos; Carlota Palma Estrada; Janet Paola Emén Sánchez;

Manuel Bernardo Balladares Mazzini

\section{RESUMEN}

Introducción: La nutrición es un proceso sistemático que involucra principalmente el rol metabólico de la célula, que es la transformación de la materia en energía, por tanto, va a influir sustancialmente en el desarrollo del individuo, y de ello el tejido cerebral es uno de los más susceptibles a las deficiencias nutricionales, especialmente, en el infante. Por lo tanto, la alteración de este proceso, va a repercutir negativamente en el rendimiento académico de los niños y niñas. En el Centro de Salud "Luchadores del Norte" de la ciudad de Guayaquil, Ecuador, se realizó una investigación que analizó el estado nutricional de escolares y su rendimiento académico. Objetivo: determinar la influencia del estado nutricional en el rendimiento escolar de los niños de Educación Básica Media, en el año 2016. Metodología: de una población de 478 estudiantes, se analizó una muestra de 193 escolares, sujetos a criterios de selección. El estudio tuvo enfoque cuantitativo, no experimental, de corte transversal, descriptivo, analítico y correlacional, el método utilizado fue la observación. Resultados: el análisis estadístico demostró que existe una asociación estadísticamente significativa entre el bajo rendimiento escolar y malnutrición, tanto en exceso como en déficit en conjunto; además, con un índice de confianza del $95 \%$ se encontró asociación entre buen rendimiento escolar y estado nutricional adecuado. Contrario a lo esperado, no se encontró asociación específica entre desnutrición crónica y bajo rendimiento. Discusión y conclusiones: el estado nutricional adecuado tiene influencia positiva en el rendimiento cognitivo de los escolares.

Palabras clave: Rendimiento académico; Desarrollo cognitivo; Desnutrición; Escolares 


\section{Influencia del estado nutricional en el rendimiento escolar de estudiantes de Educación General Básica Media}

Vol. 3, núm. 4., (2019)

Lorena Valverde Palma; Erick Reyes Ramos; Carlota Palma Estrada; Janet Paola Emén Sánchez; Manuel Bernardo Balladares Mazzini

\section{ABSTRACT}

Introduction: Nutrition is a systematic process that mainly involves the metabolic role of the cell, which we can define as the transformation of matter into energy, therefore, it will influence the entire development of the individual, and the brain tissue is one of the most susceptible tissue of nutritional deficiencies, especially in the infant. Therefore, the alteration of this process, will affect negatively in the academic performance of the boys and girls. At the "Luchadores Del Norte" Health Center in the city of Guayaquil, Ecuador, an investigation was carried to the main purpose of analyzed the nutritional status of schoolchildren and their academic performance. Objective: Determine the influence of nutritional status on school performance of children in Basic Education in the year 2016. Methodology: Population of 478 students, a sample of 193 students, subject to selection criteria, was analyzed. The study had a quantitative, nonexperimental cross-sectional, descriptive, analytical and correlational approach, the method used was observation. Results: Statistical analysis showed that there is a statistically significant association between low school performance and malnutrition which involve excess and the low intake overall; in addition, a 95\% confidence index found an association between good school performance and adequate nutritional status. Contrary to expectations, no specific association was found between chronic malnutrition and poor performance. Discussion and conclusions: Adequate nutritional status has a positive influence on the cognitive performance of schoolchildren.

Key Words: Academic performance; Cognitive development; Malnutrition; Schooling 


\section{Influencia del estado nutricional en el rendimiento escolar de estudiantes de Educación General Básica Media}

Vol. 3, núm. 4., (2019) Lorena Valverde Palma; Erick Reyes Ramos; Carlota Palma Estrada; Janet Paola Emén Sánchez; Manuel Bernardo Balladares Mazzini

\section{Introducción.}

La nutrición infantil está influenciada por varios factores como son educación de la comunidad, costumbres ancestrales, acceso a información por consejería en las unidades de salud, acceso a los alimentos, poder adquisitivo dependiente del nivel de desarrollo de los pueblos, ya que el desempleo conlleva a riesgo de inseguridad alimentaria. Existen otras condiciones de riesgo asociadas a desnutrición crónica o fallo de medro desde etapas tempranas en la infancia y que se mantienen por años, entre ellas la estructura familiar incompleta, que predispone a disfunción en el hogar del infante. ${ }^{1}$ Por otro lado, la nutrición infantil está directamente relacionada con el desarrollo físico y neurodesarrollo desde el nacimiento, lo cual implica adquisición de pautas madurativas, que en la etapa escolar están relacionadas con la actividad cognitiva del individuo. La desnutrición infantil, presente durante largos periodos, va a tener impacto adverso en el neurodesarrollo de niños y niñas, y a largo plazo, desencadenará un bajo rendimiento escolar. ${ }^{2}$

A nivel mundial, más de un cuarto (26\%) de los niños menores de 5 años con desnutrición crónica (alrededor 165 millones de niños en el mundo), en el 2011 estuvo distribuida principalmente en África subsahariana y Asia meridional, con mayor afectación en India, Nigeria y Pakistán. ${ }^{3}$ La crisis económica en España tuvo repercusiones negativas en la alimentación como en la trayectoria educativa de los niños. ${ }^{4}$

El crecimiento infantil es dinámico, con picos y mesetas, e influido por factores genéticos, ambientales, socioeconómicos, culturales-alimentarios. $5,6,7$ En Ecuador, la desnutrición crónica infantil en menores de 6 años, ha evolucionado, con tasas del 40,2\% en 


\section{Influencia del estado nutricional en el rendimiento escolar de estudiantes de Educación General Básica Media}

Vol. 3, núm. 4., (2019)

Lorena Valverde Palma; Erick Reyes Ramos; Carlota Palma Estrada; Janet Paola Emén Sánchez; Manuel Bernardo Balladares Mazzini

1986, 33,3\% en 2004, y 25,2\% en 2012. Las cifras de desnutrición en escolares están en el rango $14,6 \%$ a $15,8.8,9$

El desarrollo cerebral inicia a las pocas semanas de edad gestacional, durante la primera infancia continúa la mielinización. Existe un patrón propio con brotes de crecimiento del tejido cerebral, periodos sensibles, en los cuales, las influencias ambientales van a causar efectos en el desarrollo y expresión génica. ${ }^{10,}{ }^{11} \mathrm{El}$ desarrollo cognoscitivo en lactantes corresponde al desarrollo sensorio motriz a partir de la experiencia e imitación. En preescolares inicia el pensamiento lógico, basado en la investigación, aprendizaje y adaptación. En escolares se inicia la construcción del pensamiento concreto, predomina el pensamiento inductivo y deductivo, a través de la solución de problemas de manera lógica, lo que constituye la base del aprendizaje. ${ }^{12}$, 13,14

La valoración nutricional antropométrica, se realiza a partir de los patrones de referencia de la OMS, ${ }^{15}$ que, asociado a los demás componentes como la historia clínica dietética, valoración subjetiva global, estudios bioquímicos, nos permiten obtener una perspectiva global del estado nutricional del infante. ${ }^{16}$

El rendimiento académico es evidenciado con las calificaciones obtenidas en las diferentes áreas de aprendizaje, a través de escalas cuantitativas, con su equivalente cualitativo. ${ }^{17}$,

${ }^{18}$ En nuestro país, la educación general básica media (5o, 6o, 7o año), evidencia objetivamente la calificación, y es cuando los padres sienten preocupación por el rendimiento académico de sus hijos y buscan remediar la situación, incluyendo la nutrición si es parte del problema. 


\section{Influencia del estado nutricional en el rendimiento escolar de estudiantes de Educación General Básica Media}

Vol. 3, núm. 4., (2019) Lorena Valverde Palma; Erick Reyes Ramos; Carlota Palma Estrada; Janet Paola Emén Sánchez;

Manuel Bernardo Balladares Mazzini

Hay estudios que demuestran asociación significativa entre desnutrición infantil y menor desempeño intelectual; ${ }^{19,20,21}$ además, se ha encontrado afectación de los procesos de atención en adulto, que tuvieron desnutrición en la infancia, a pesar de haber recibido rehabilitación nutricional, lo que confirma lo grave e irreversible de los efectos de la desnutrición en el desarrollo cognoscitivo. ${ }^{22}$ Otros estudios asocian la desnutrición crónica con parasitosis, déficit de micronutrientes como hierro, y bajo desarrollo cognitivo. ${ }^{23}$ En Tanzania la desnutrición crónica leve a severa también se asoció con mayores déficits del neurodesarrollo en niños, y los infantes con desnutrición aguda, presentaron retrasos durante la etapa de carencia. ${ }^{21,24}$

La implementación de esquemas de financiación innovadores en salud, especialmente en los países pobres, donde la comunidad sea co-responsable, especialmente en el ámbito de la nutrición infantil, muestran resultados alentadores, ${ }^{25}$ por ello, los factores de riesgo asociados a las alteraciones del desarrollo cognitivo, deben ser intervenidos de manera prioritaria, especialmente los alimentarios, para favorecer la calidad de vida de los niños afectados. ${ }^{26}$

En la ciudad de Guayaquil, Ecuador, se realizó un estudio, cuyo el objetivo fue determinar la influencia del estado nutricional en el rendimiento académico de los niños de Educación Básica Media, atendidos en el programa de Salud Escolar del centro de Salud "Luchadores del Norte", año 2016. Los resultados demostraron asociación significativa entre malnutrición infantil, que incluye el sobrepeso, la obesidad y la desnutrición, con bajo rendimiento escolar; además, con una probabilidad del 95\% se encontró asociación entre buen rendimiento escolar y estado nutricional adecuado. Contrario a lo esperado, no se encontró 


\section{Influencia del estado nutricional en el rendimiento escolar de estudiantes de Educación General Básica Media}

Vol. 3, núm. 4., (2019)

Lorena Valverde Palma; Erick Reyes Ramos; Carlota Palma Estrada; Janet Paola Emén Sánchez; Manuel Bernardo Balladares Mazzini

asociación entre desnutrición crónica y bajo rendimiento; por lo tanto, se concluye que el estado nutricional adecuado tiene influencia positiva en el rendimiento cognitivo de los escolares.

Materiales y Métodos.

La metodología empleada tiene un enfoque cuantitativo, con asociación o relación entre las variables, de diseño no experimental, corte transversal; el método es observacional, el tipo de estudio es analítico y correlacional. Para el análisis bibliográfico se utilizaron varias fuentes documentales, realizándose una búsqueda en meta buscadores como Pubmed, Medline, Cochrane, Scielo, utilizando como descriptores las palabras claves. Esta búsqueda se realizó de septiembre del 2016 a marzo del 2017.

Población: Estuvo conformado por 540 estudiantes de educación básica de la Escuela “Jorge Enrique Adoum” incluidos en el Programa de Control Escolar del Centro de Salud "Luchadores del Norte"

Muestra: Estuvo constituida por los 193 estudiantes de educación general básica media, del Programa de Control Escolar del Centro de Salud "Luchadores del Norte" y Escuela "Jorge Enrique Adoum", sujeta a criterios de selección

Instrumentos de evaluación o recolección de la data: Para la recopilación de la información se diseñó una Ficha de Recolección de Datos, la misma que se sustentó en la Historia Clínica Institucional del Control de Salud Escolar. 


\section{Influencia del estado nutricional en el rendimiento escolar de estudiantes de Educación General Básica Media}

Vol. 3, núm. 4., (2019) Lorena Valverde Palma; Erick Reyes Ramos; Carlota Palma Estrada; Janet Paola Emén Sánchez; Manuel Bernardo Balladares Mazzini

El Diagnóstico del Estado Nutricional en niños y niñas escolares, se realizó en base al Estudio Multicéntrico de la OMS sobre Patrones de Crecimiento Infantil, que son la referencia que orientan este diagnóstico de manera objetiva y adecuada. ${ }^{27}$ Se establecieron los índices o coeficientes, siendo los más utilizados:

El índice Pesoledad, o índice Nutricional de Gómez, ${ }^{28}$ que se calcula dividiendo el Peso Actual para el Peso correspondiente a esa edad, en percentil 50, y multiplicado por 100 [( $\mathrm{P}_{\text {actual }} /$ $\left.P_{\text {ideal }}\right)$ 100], los puntos de corte son:

Mayor al 120\%: $\quad$ Obesidad

110-120\%: $\quad$ Sobre Peso

91 a $110 \%$ : $\quad$ Normopeso - Eutrófico

76 a 90 \%: $\quad$ Desnutrición Grado I o Leve

61 a $76 \%$ : $\quad$ Desnutrición Grado II o Moderada

Menor a $61 \% \quad$ Desnutrición Grado III o Severa

En lo que se refiere a Talla por Edad (T/E), es el reflejo del crecimiento vertical y se ve modificado ante carencias nutricionales de larga data, ${ }^{29}$ sus puntos de corte son:

Menor al Percentil 3: Desnutrición crónica, Talla Baja, Retardo en el Crecimiento

Percentil 3 a 15: $\quad$ Riesgo de talla baja 


\section{Influencia del estado nutricional en el rendimiento escolar de estudiantes de Educación General Básica Media}

Vol. 3, núm. 4., (2019)

Lorena Valverde Palma; Erick Reyes Ramos; Carlota Palma Estrada; Janet Paola Emén Sánchez; Manuel Bernardo Balladares Mazzini

Mayor al Percentil 15: Talla normal

El IMC se calcula al dividir el Peso en Kilogramos por la estatura en metros elevado al cuadrado $\left[\mathrm{P}(\mathrm{Kg}) / \mathrm{T}\left(\mathrm{m}^{2}\right)\right]$, y los puntos de corte son: ${ }^{27,30}$
Menor o igual al Percentil 3:
Desnutrición

Percentil 3 a 15:

Bajo Peso o riesgo de desnutrición

Pc 15 a 85:

Normopeso o eutrófico

Pc 85 a 97:

Sobrepeso

Mayor o igual al Percentil 97:

\section{Obesidad}

La valoración académica promedio sustentada en la norma del Ministerio de Educación, con su equivalente numérico, fue obtenida de los registros escolares, y sus parámetros son:

\section{Escala cualitativa}

Supera los aprendizajes requeridos (Muy Satisfactorio)

Domina los aprendizajes requeridos (Satisfactorio)

Alcanza los aprendizajes requeridos (Mejorable)

Está próximo a alcanzar los aprendizajes requeridos (Poco Satisfactorio) 5-6

No alcanza los aprendizajes requeridos (Insatisfactorio)

\section{Escala cuantitativa}

10

9

$7-8$

$\leq 4$ 


\section{Influencia del estado nutricional en el rendimiento escolar de estudiantes de Educación General Básica Media}

Vol. 3, núm. 4., (2019) Lorena Valverde Palma; Erick Reyes Ramos; Carlota Palma Estrada; Janet Paola Emén Sánchez; Manuel Bernardo Balladares Mazzini

A partir de esta escala se consideró como bajo rendimiento escolar a los puntajes correspondientes a mejorable, poco satisfactorio e insatisfactorio, y buen rendimiento escolar a satisfactorio y muy satisfactorio.

Posteriormente se aplicó la Ficha de manera individual donde se anotaron los datos relacionados con el estudio a partir de la Historia Clínica, simultáneamente al realizar el control escolar. Además, se diseñó una base de datos que consolidó la información de los casos de estudio, y se procedió al análisis estadístico correspondiente.

\section{Metodología para el análisis de los resultados}

Una vez compilada la información en la base de datos, se procedió a análisis de la misma, para lo cual se utilizó el programa SPSS versión 22. Inicialmente se realizó el análisis de estadística descriptiva, como frecuencias relativas y absolutas, y medidas de tendencia central, para luego proceder al análisis de asociación estadística para pruebas de significancia, como lo es la prueba del Chi cuadrado, a partir del cual se realizó la comprobación de hipótesis.

\section{Consideraciones biotécnicas}

En el presente estudio, los aspectos bioéticos considerados fueron la confidencialidad de la información, y el respeto a la integridad de los individuos. La ejecución del Proyecto no involucró intervención en el ámbito personal, familiar o comunitario, además tuvo las autorizaciones de los representantes de la Unidad de Salud del Ministerio de Salud Pública, y de los Directivos de la Unidad Educativa. Se trató de una investigación sin riesgo, ya que no hubo 


\section{Influencia del estado nutricional en el rendimiento escolar de estudiantes de Educación General Básica Media}

Vol. 3, núm. 4., (2019)

Lorena Valverde Palma; Erick Reyes Ramos; Carlota Palma Estrada; Janet Paola Emén Sánchez; Manuel Bernardo Balladares Mazzini

manipulación de los sujetos de estudio, los cuales fueron incluidos en el trabajo de acuerdo a criterios de selección.

\section{Resultados.}

Al término del estudio, se hallaron los siguientes resultados: La frecuencia por edad fue de 83 casos (43\%) entre 9-10 años, 98 casos (50,8\%) entre 11-12 años, y 12 casos (6,2\%) mayores de 12 años. La frecuencia por sexo mostró un leve predominio del masculino con 104 casos $(53.9 \%)$, sobre el femenino 89 casos $(46.1 \%)$.

Los parámetros antropométricos tuvieron las siguientes medidas de tendencia central: el Peso una media de 40.00, mediana 38 y DS 10.83; la Talla una media de 1.39 , mediana 1.39 y DS 0.09; el IMC una media de 20.18, mediana 19.20 y DS 3.95. Las frecuencias por año de Educación Básica, fueron: $5^{\circ}$ año 68 casos (35.2\%), $6^{\circ}$ año 60 casos $(31.3 \%), 7^{\circ}$ año 65 casos (33.7\%), con una distribución uniforme.

A partir del índice peso/edad se realizó el Diagnóstico nutricional, siendo más frecuente el correspondiente a sobrepeso y obesidad con 90 casos (46,6\%), eutrófico 49 casos (25.4\%), y desnutrición 54 casos $(28 \%)$ asociada a mayor frecuencia de talla baja, con lo que se evidencia que nuestra población infantil tiene malnutrición, con mayor tendencia al exceso de peso, y un menor porcentaje sigue un patrón de crecimiento normal. (Figura 1)

Figura 1. Distribución relativa y absoluta del Diagnóstico Nutricional en función del Índice (Porcentaje) Peso-Edad 


\section{Influencia del estado nutricional en el rendimiento escolar de estudiantes de Educación General Básica Media}

Vol. 3, núm. 4., (2019) Lorena Valverde Palma; Erick Reyes Ramos; Carlota Palma Estrada; Janet Paola Emén Sánchez;

Manuel Bernardo Balladares Mazzini

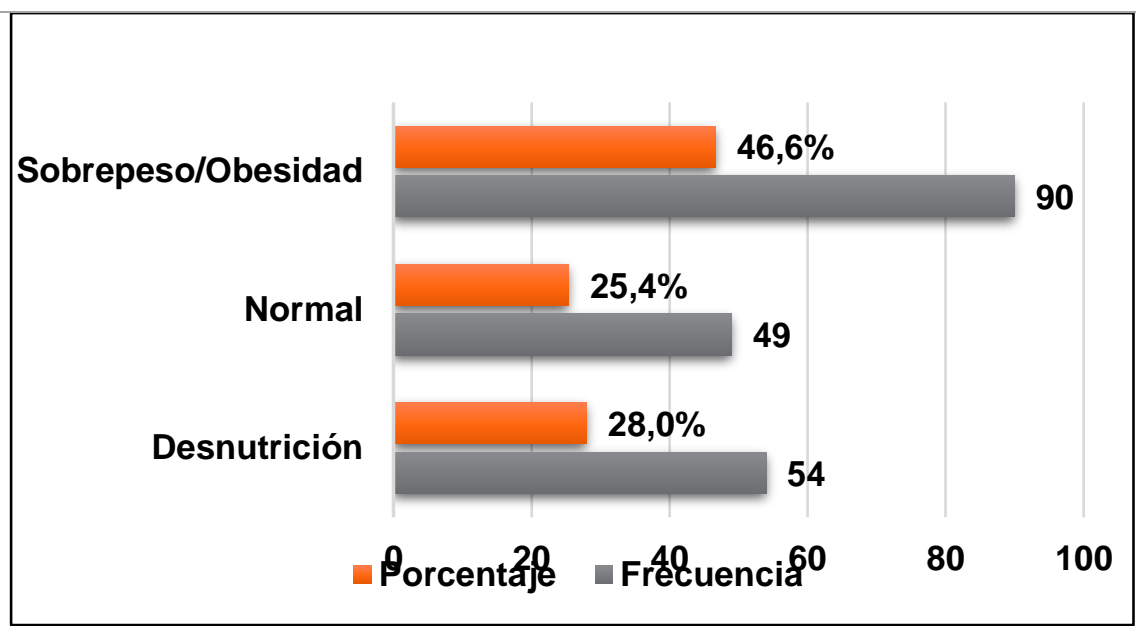

Fuente: Base de datos

Elaborado por: Autores

El IMC tuvo una distribución que marcó un claro predominio de sobrepeso y obesidad con 41 casos $(21.2 \%)$ y 51 casos $(26.4 \%)$ respectivamente, el percentil 15-85 de normalidad tuvo 88 casos (45.6\%) y la desnutrición que incluye P3-P15 y menor al P3, presentó 11 (5.75\%), y 2 casos $(1 \%)$ respectivamente, pero los percentiles de normalidad, sobrepeso y obesidad estuvieron asociados a talla baja, por lo que, este indicador no fue considerado útil como diagnóstico nutricional para ser relacionado con el rendimiento escolar. (Tabla 1).

Tabla 1: Tabulación cruzada del Percentil de Talla con el Índice (Porcentaje) Peso/Edad y

Percentil de IMC

\begin{tabular}{|c|c|c|c|c|c|c|c|c|c|c|c|}
\hline \multirow{3}{*}{\multicolumn{2}{|c|}{ PERCENTIL $\leq \mathrm{P} 3$}} & \multicolumn{3}{|c|}{$\%$ PESO/EDAD } & \multirow[b]{2}{*}{ Total } & \multicolumn{5}{|c|}{ PERCENTIL IMC } & \multirow[t]{2}{*}{ Total } \\
\hline & & $\leq 90$ & $91-110$ & $>110$ & & $\leq \mathrm{P} 3$ & P3-15 & P15-85 & P85-97 & $\geq \mathrm{P} 97$ & \\
\hline & & 16 & 8 & 4 & 28 & 0 & 3 & 16 & 5 & 4 & 28 \\
\hline DE & P3-P15 & 23 & 9 & 17 & 49 & 2 & 4 & 26 & 6 & 11 & 49 \\
\hline
\end{tabular}




\section{Influencia del estado nutricional en el rendimiento escolar de estudiantes de Educación General Básica Media}

Vol. 3, núm. 4., (2019)

Lorena Valverde Palma; Erick Reyes Ramos; Carlota Palma Estrada; Janet Paola Emén Sánchez; Manuel Bernardo Balladares Mazzini

\begin{tabular}{|l|l|l|l|l|l|l|l|l|l|l|}
$>\mathrm{P} 15$ & 15 & 32 & 69 & 116 & 0 & 4 & 46 & 30 & 36 & 116 \\
Total & 54 & 49 & 90 & 193 & 2 & 11 & 88 & 41 & 51 & 193 \\
\hline
\end{tabular}

Fuente: Base de datos

Elaborado por: Autores

La cronicidad de la desnutrición, se valora a través del índice talla/edad, encontrándose desnutrición crónica o retraso en el crecimiento (Talla baja) en 28 casos (14,5\%), riesgo de desnutrición crónica en 49 casos (26\%), y Talla normal 116 casos $(60.1 \%)$, lo cual coincide con los parámetros de la encuesta ENSANUT-Ecu del 2013. (Figura 2).

Figura 2. Distribución relativa y absoluta del Diagnóstico de Retraso del Crecimiento o Desnutrición Crónica (Talla Baja)



Fuente: Base de datos

Elaborado por: Autores 


\section{Influencia del estado nutricional en el rendimiento escolar de estudiantes de Educación General Básica Media}

Vol. 3, núm. 4., (2019) Lorena Valverde Palma; Erick Reyes Ramos; Carlota Palma Estrada; Janet Paola Emén Sánchez; Manuel Bernardo Balladares Mazzini

El rendimiento escolar estuvo considerado para este estudio como Muy satisfactorio 13 casos $(6,7 \%)$, Satisfactorio 65 casos $(33,7 \%)$, mejorable 98 casos $(50,8 \%)$ y poco satisfactorio 17 casos $(8,8 \%)$, utilizando para ello el promedio de notas del primer quimestre del 2016, de las materias de Matemáticas, Lenguaje, Ciencias Sociales y Ciencias Naturales. El promedio para mejorable y poco satisfactorio es equivalente a la nota igual o menor a 8; y, según el Ministerio de Educación, es el niño que solo alcanza los aprendizajes requeridos o está próximo a ello, constituyéndose en rendimiento escolar bajo (Figura 3).

Figura 3. Distribución relativa y absoluta del Rendimiento Escolar



Fuente: Base de datos

Elaborado por: Autores 


\section{Influencia del estado nutricional en el rendimiento escolar de estudiantes de Educación General Básica Media}

Vol. 3, núm. 4., (2019)

Lorena Valverde Palma; Erick Reyes Ramos; Carlota Palma Estrada; Janet Paola Emén Sánchez; Manuel Bernardo Balladares Mazzini

Se evaluó también factores de riesgo consignados en la Historia clínica, siendo los más relevantes los relacionados con la alimentación, morbilidades del niño y estructura familiar, encontrándose predomino de la estructura familiar afectada en 60 casos (31\%).

Se realizó prueba de hipótesis, contrastando el diagnóstico nutricional con el rendimiento académico: De las hipótesis planteadas, se encontró asociación estadísticamente significativa entre bajo rendimiento escolar y malnutrición, que incluye sobrepeso/obesidad y desnutrición. El P valor fue de 0,040 , menor a 0,05 , por lo que se rechaza la hipótesis nula, se concluye con el 95\% de confianza que existe evidencia estadística para rechazar independencia entre las variables (Tabla 2).

Tabla 2: Prueba de chi-cuadrado: Asociación entre las variables Bajo Rendimiento Escolar y

$$
\text { Malnutrición }
$$

\begin{tabular}{c|ccccc}
\hline & Valor & gl & $\begin{array}{c}\text { Sig. asintótica (2 } \\
\text { caras) }\end{array}$ & $\begin{array}{c}\text { Significación exacta (2 } \\
\text { caras) }\end{array}$ & $\begin{array}{c}\text { Significación exacta (1 } \\
\text { cara) }\end{array}$ \\
\hline Chi-cuadrado de Pearson & 4,197 & 1 & 0,040 & & \\
Corrección de continuidad & 3,526 & 1 & 0,060 & 0,042 & 0,029 \\
Razón de verosimilitud & 4,343 & 1 & 0,037 & & \\
Prueba exacta de Fisher & & & & & \\
$\quad$ N de casos válidos & 193 & & & \\
\hline
\end{tabular}

Fuente: Base de datos

\section{Elaborado por: Autores}

Análisis: Con un índice de confianza del 95\% se encuentra asociación significativa entre las variables, ya que el $\mathrm{P}$ valor es menor de 0,05 , lo que indica rechazar la hipótesis nula, y 


\section{Influencia del estado nutricional en el rendimiento escolar de estudiantes de Educación General Básica Media}

Vol. 3, núm. 4., (2019) Lorena Valverde Palma; Erick Reyes Ramos; Carlota Palma Estrada; Janet Paola Emén Sánchez;

Manuel Bernardo Balladares Mazzini

aceptar la hipótesis alterna, que demuestra la existencia de una relación entre bajo rendimiento escolar y malnutrición.

La desnutrición infantil crónica, retraso del crecimiento, o talla baja, no estuvo relacionada con el bajo rendimiento escolar de la muestra estudiada, ya que la prueba de chi cuadrado dio un $\mathrm{P}$ valor de 0,700 mayor a 0,05, por lo cual se acepta la hipótesis nula que indica independencia entre variables.

La prueba de chi cuadrado, demostró una asociación estadísticamente significativa entre las variables estado nutricional adecuado y buen rendimiento escolar, ya que el $\mathrm{P}$ valor fue 0,040, menor de 0,05 , por lo que se rechaza la hipótesis nula. Se concluye con el $95 \%$ de confianza que existe evidencia estadística para rechazar independencia entre las variables.

\section{Discusión}

Al analizar los resultados del estudio, sobre el estado nutricional de la población escolar, en lo que respecta a la desnutrición crónica o retraso del crecimiento, es coincidente con la encuesta ENSANUT-Ecu del 2013, con los estudios realizados en Bolivia en el 2016, y en Perú en el 2013. ${ }^{8,19,}{ }^{20}$ Los indicadores de sobrepeso y obesidad son similares a los hallados en Colombia en el 2013. ${ }^{14}$

En cuanto a la asociación entre las variables de desnutrición y retraso en el crecimiento con el rendimiento escolar, no se logró establecer la misma con las pruebas de significancia estadística, lo que indica que existe independencia entre variables, lo cual no es concordante con los hallazgos de Galler en Barbados el 2012, o Binagwaho en Ruanda el 2014, Sudfeld en el 


\section{Influencia del estado nutricional en el rendimiento escolar de estudiantes de Educación General Básica Media}

Vol. 3, núm. 4., (2019)

Lorena Valverde Palma; Erick Reyes Ramos; Carlota Palma Estrada; Janet Paola Emén Sánchez; Manuel Bernardo Balladares Mazzini

metaanálisis realizado el 2015, y Sudfeld en Tanzania el 2015, donde hallaron una asociación significativa entre desnutrición crónica y desarrollo cognitivo. ${ }^{21,22,24,25}$

Los resultados concuerdan con el estudio de Roldán González en Colombia el 2013, donde no hubo asociación entre sobrepeso y obesidad con el rendimiento académico. ${ }^{14}$ Pero al analizar la variable como malnutrición que incluye sobrepeso/obesidad y desnutrición, con bajo rendimiento escolar, se encontró una probabilidad de $95 \%$ de que estas variables estén asociadas.

Las condiciones de riesgo como la estructura familiar, tratándose en muchos casos de familias incompletas en el estudio, contribuye al bajo rendimiento escolar; este factor se relaciona con los hallazgos de Fernández en Cuba, en el cual los factores familiares están relacionados con la desnutrición crónica o falla de medro en lactantes, ${ }^{1}$ esto último directamente asociado a afectación del desarrollo cognitivo.

\section{Conclusiones.}

El estado nutricional de los estudiantes de EGB, atendidos en el Programa de salud Escolar del CS “Luchadores del Norte”, sigue un patrón de crecimiento igual al de la población infantil general en Ecuador, con una clara tendencia a la obesidad, pero aún persiste la desnutrición crónica en cifras estacionadas, así como una frecuencia importante de niños con riesgo de desnutrición crónica, en una etapa donde se establece la talla definitiva.

Se halló una clara tendencia hacia el bajo rendimiento académico de los estudiantes de EGB. 


\section{Influencia del estado nutricional en el rendimiento escolar de estudiantes de Educación General Básica Media}

Vol. 3, núm. 4., (2019) Lorena Valverde Palma; Erick Reyes Ramos; Carlota Palma Estrada; Janet Paola Emén Sánchez; Manuel Bernardo Balladares Mazzini

Existe una asociación significativa entre malnutrición en general, constituida por sobrepeso-obesidad y desnutrición, con el rendimiento académico de los escolares en estudio. No se demostró asociación entre desnutrición aislada y rendimiento escolar, pero hay una alta frecuencia de bajo rendimiento en niños con desnutrición crónica.

El estado nutricional normal influye de manera positiva en el rendimiento académico de los escolares.

\section{Bibliografía.}

1. Fernández F, Lourdes M de, Borrego P, Enrique C, Rodríguez P, Karla L. Algunos factores familiares asociados a fallo de medro en lactantes. Rev Cuba Med Gen Integral. 28 de septiembre de 2015;31(3):333-45.

2. Martorell R, Horta BL, Adair LS, Stein AD, Richter L, Fall CHD, et al. Weight Gain in the First Two Years of Life Is an Important Predictor of Schooling Outcomes in Pooled Analyses from Five Birth Cohorts from Low- and Middle-Income Countries. J Nutr. 2 de enero de 2010;140(2):348-54.

3. United Nations International Children's Fund. Improving Child Nutrition: The achievable imperative for global progress. En: United Nations Children's Fund (UNICEF) [Internet]. New York, USA; 2013 [citado 23 de mayo de 2017]. p. 7-16. Disponible en: https://www.unicef.org/publications/files/Nutrition_Report_final_lo_res_8_April.pdf

4. González-Bueno G. Pobreza infantil e impacto de la crisis en la infancia. Educ Futuro Rev Investig Apl Exp Educ. 2014;(30):109-26.

5. Briceño G, Durán P, Colón E, Line D, Merker A, Abad V, et al. Protocolo del estudio para establecer estándares normativos de crecimiento de niños colombianos sanos. Pediatría. Diciembre de 2012;45(4):235-42.

6. Marcdante KJ, Behrman RE, Jenson HB. Crecimiento normal. In KJ, Behrman RE, Jenson HB. Nelson. En: Pediatría esencial. 7th ed. Barcelona: Elsevier; 2015. p. 10-26.

7. Kliegman R, Stanton B, St. Geme J, Schor N, Behrman R. Crecimiento, desarrollo y conducta. En: Nelson Tratado de Pediatría. Madrid: Elsevier; 2012. 


\section{Influencia del estado nutricional en el rendimiento escolar de estudiantes de Educación General Básica Media}

Vol. 3, núm. 4., (2019)

Lorena Valverde Palma; Erick Reyes Ramos; Carlota Palma Estrada; Janet Paola Emén Sánchez; Manuel Bernardo Balladares Mazzini

8. Freire W, Ramírez-Luzuriaga J, Belmont P, Mendieta MJ, Silva-Jaramillo K, Romero N, et al. Estado Nutricional a partir de indicadores antropométricos. Encuesta Nacional de Salud y Nutrición - ENSANUT - Ministerio de Salud Pública [Internet]. [citado 5 de abril de 2017]. Disponible en: http://www.salud.gob.ec/encuesta-nacional-de-salud-y-nutricionensanut/

9. Sistema de Vigilancia Alimentario Nutricional. Reportes SIVAN 2014 [Internet]. QuitoEcuador: Ministerio de Salud Pública; 2014 p. 1. Disponible en: http://www.salud.gob.ec/unidad-de-nutricion/

10. Kang HJ, Kawasawa YI, Cheng F, Zhu Y, Xu X, Li M, et al. Spatio-temporal transcriptome of the human brain. Nature. 27 de octubre de 2011;478(7370):483-9.

11. Oates J, Karmiloff-Smith A, Johnson MH. Sensitive periods. En: Developing Brains [Internet]. United Kingdom: The Open University; 2012. p. 28-32. (EARLY CHILDHOOD IN FOCUS Series edited by Martin Woodhead and John Oates). Disponible en: http://oro.open.ac.uk/33493/1/Developing_Brains.pdf

12. Bastidas Acevedo M, Posada Díaz Á, Ramírez Gómez H. Crecimiento y desarrollo del niño preescolar - Crecimiento y desarrollo del niño escolar. En: El Niño Sano [Internet]. 3a ed. Bogotá: Ed. Médica Panamericana; 2005. p. 148-57. Disponible en: https://books.google.es/books?id=k0wodPSaT4cC\&pg=PA3\&hl=es\&source=gbs_selecte $\mathrm{d} \_$pages $\&$ cad $=2 \# \mathrm{v}=$ onepage $\& \mathrm{q} \& \mathrm{f}=$ false

13. Pérez Jácome A, Mena Gutiérrez Hernández G, Vela Gutiérrez G, Flores Guillén LE, López Pérez AL de J. Efectos de la malnutrición en el aprendizaje y rendimiento escolar en niños preescolares en Tuxtla Gutiérrez, Chiapas, México. Lacandonia. 16 de mayo de 2017;6(1):99-102.

14. Roldán González E, Paz Ortega A. Relación de sobrepeso y obesidad con nivel de actividad física, condición física, perfil psicomotor y rendimiento escolar en población infantil (8 a 12 años) de Popayán. Rev Mov Científico. 31 de diciembre de 2013;7(1):7184.

15. Organización Mundial de la Salud. Patrones de crecimiento infantil de la OMS.

16. Hammond K. Ingesta: análisis de la dieta. En: Krause Dietoterapia. 13a ed. Barcelona España: ELSEVIER; 2013. p. 129-42.

17. Ministerio de Educación. Instructivo para la aplicación de la Evaluación Estudiantil [Internet]. 2013. Disponible en: https://educacion.gob.ec/wpcontent/uploads/downloads/2013/10/Instructivo_para_evaluacion_estudiantil_2013.pdf 


\section{Influencia del estado nutricional en el rendimiento escolar de estudiantes de Educación General Básica Media}

Vol. 3, núm. 4., (2019)

Lorena Valverde Palma; Erick Reyes Ramos; Carlota Palma Estrada; Janet Paola Emén Sánchez;

Manuel Bernardo Balladares Mazzini

18. Rojas Guerrero NF, Li Loo Kung CA, Dávila Panduro SK, Alva Angulo MR. El estado nutricional y su impacto en los logros de aprendizaje. Cienc Amaz Iquitos. 30 de diciembre de 2015;5(2):115-20.

19. Pajuelo-Ramírez J, Sánchez-Abanto J, Alvarez-Dongo D, Tarqui-Mamani C, AgüeroZamora R. Sobrepeso, obesidad y desnutrición crónica en niños de 6 a 9 años en Perú, 2009-2010. Rev Peru Med Exp Salud Publica. octubre de 2013;30(4):583-9.

20. Lafuente Y. KV, Rodriguez S, Fontaine V, Yañez V. R. Prevalencia de la desnutrición crónica en niños menores de 5 años atendidos en el Centro de Salud Tacopaya, primer semestre gestión 2014. Gac Médica Boliv. junio de 2016;39(1):26-9.

21. Sudfeld CR, McCoy DC, Danaei G, Fink G, Ezzati M, Andrews KG, et al. Linear Growth and Child Development in Low- and Middle-Income Countries: A Meta-Analysis. Pediatrics. 1 de mayo de 2015;135(5):e1266-75.

22. Galler J, Bryce C, Zichlin M, Fitzmaurice G, Eaglesfield D, Waber D. Infant Malnutrition Is Associated with Persisting Attention Deficits in Middle Adulthood. abril de 2012;142(4):788-94.

23. Perignon M, Fiorentino M, Kuong K, Burja K, Parker M, Sisokhom S, et al. Stunting, Poor Iron Status and Parasite Infection Are Significant Risk Factors for Lower Cognitive Performance in Cambodian School-Aged Children. PLOS ONE. 18 de noviembre de 2014;9(11): 112605 .

24. Sudfeld CR, McCoy DC, Fink G, Muhihi A, Bellinger DC, Masanja H, et al. Malnutrition and Its Determinants Are Associated with Suboptimal Cognitive, Communication, and Motor Development in Tanzanian Children. J Nutr. 12 de enero de 2015;145(12):270514.

25. Binagwaho A, Condo J, Wagner C, Ngabo F, Karema C, Kanters S, et al. Impact of implementing performance-based financing on childhood malnutrition in Rwanda. BMC Public Health. 2014;14: 1132.

26. Walker SP, Wachs TD, Grantham-McGregor S, Black MM, Nelson CA, Huffman SL, et al. Inequality in early childhood: risk and protective factors for early child development. The Lancet. 8 de octubre de 2011;378(9799):1325-38.

27. Pacheco G, Pernas G, Mosqueira M, Juiz de Trogliero C, Raineri F, Piazza N. Evaluación del crecimiento de niños y niñas. Nuevas referencias de la Organización Mundial de la Salud (OMS). En: Evaluación del crecimiento de niños y niñas [Internet]. Primera. SaltaArgentina: unicef; 2012. p. 7-31. Disponible en: https://www.unicef.org/argentina/spanish/Nutricion_24julio.pdf 


\section{Influencia del estado nutricional en el rendimiento escolar de estudiantes de Educación General Básica Media}

Vol. 3, núm. 4., (2019)

Lorena Valverde Palma; Erick Reyes Ramos; Carlota Palma Estrada; Janet Paola Emén Sánchez; Manuel Bernardo Balladares Mazzini

28. Márquez-González H, García-Sámano VM, Caltenco-Serrano M de L, García-Villegas EA, Márquez-Flores H, Villa-Romero AR. Clasificación y evaluación de la desnutrición en el paciente pediátrico. El Resid. 2012;7(2):59-69.

29. Martínez Costa C, Pedrón Giner C. Valoración del estado nutricional. En: Protocolos diagnóstico-terapéuticos de Gastroenterología, Hepatología y Nutrición Pediátrica SEGHNP-AEP [Internet]. 2a edición. Madrid, España: Ergón S.A.; 2010. p. 313-8. Disponible en: https://www.aeped.es/sites/default/files/documentos/valoracion_nutricional.pdf

30. Braun Y, Brest F, Fernández V, Guezikaraian N. Comparación de estándares antropométricos. Rev Ciencias de la Salud. septiembre de 2011;2(1):10-20.

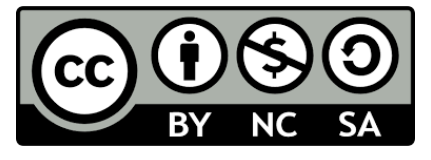

RECONOCIMIENTO-NOCOMERCIAL-COMPARTIRIGUAL

CC BY-NC-SA

ESTA LICENCIA PERMITE A OTROS ENTREMEZCLAR, AJUSTAR Y CONSTRUIR A PARTIR DE SU OBRA CON FINES NO COMERCIALES, SIEMPRE Y CUANDO LE RECONOZCAN LA AUTORÍA Y SUS NUEVAS CREACIONES ESTÉN BAJO UNA LICENCIA CON LOS MISMOS TÉRMINOS. 